\title{
Adenosine kinase deficiency: expanding the clinical spectrum and evaluating therapeutic options
}

\author{
Christian Staufner ${ }^{1} \cdot$ Martin Lindner ${ }^{1,2} \cdot$ Carlo Dionisi-Vici $^{3} \cdot$ Peter Freisinger $^{4}$. \\ Dries Dobbelaere $^{5}$ - Claire Douillard ${ }^{5}$ - Nawal Makhseed ${ }^{6}$ - Beate K. Straub ${ }^{7}$. \\ Kimia Kahrizi $^{8}$ - Diana Ballhausen ${ }^{9}$ - Giancarlo la Marca ${ }^{10} \cdot$ Stefan Kölker $^{1}$. \\ Dorothea Haas $^{1}$ • Georg F. Hoffmann ${ }^{1}$ - Sarah C. Grünert ${ }^{11}$ • Henk J. Blom ${ }^{11}$
}

Received: 1 September 2015 / Revised: 10 November 2015 / Accepted: 11 November 2015 /Published online: 7 December 2015

(C) SSIEM 2015

\begin{abstract}
Background Adenosine kinase deficiency is a recently described defect affecting methionine metabolism with a severe clinical phenotype comprising mainly neurological and hepatic impairment and dysmorphism.

Methods Clinical data of 11 additional patients from eight families with adenosine kinase deficiency were gathered through a retrospective questionnaire. Two liver biopsies of one patient were systematically evaluated.

Results The main clinical symptoms are mild to severe liver dysfunction with neonatal onset, muscular hypotonia, global developmental retardation and dysmorphism (especially frontal bossing). Hepatic involvement is not a constant finding. Most patients have epilepsy and recurrent hypoglycemia due
\end{abstract}

Communicated by: Viktor Kožich

Electronic supplementary material The online version of this article (doi:10.1007/s10545-015-9904-y) contains supplementary material, which is available to authorized users.

Christian Staufner

christian.staufner@med.uni-heidelberg.de

1 Department of General Pediatrics, Division of Pediatric Metabolic Medicine and Neuropediatrics, University Hospital Heidelberg, Im Neuenheimer Feld 430, 69120 Heidelberg, Germany

2 Department of Neurology, University Children's Hospital Frankfurt, Frankfurt, Germany

3 Division of Metabolism, Bambino Gesù Children's Hospital IRCCS, Rome, Italy

4 Children's Hospital Reutlingen, Reutlingen, Germany

5 Reference Center for Inherited Metabolic Diseases in Child and Adulthood, University Children's Hospital Jeanne de Flandre, Lille Cedex, France to hyperinsulinism. Major biochemical findings are intermittent hypermethioninemia, increased S-adenosylmethionine and Sadenosylhomocysteine in plasma and increased adenosine in urine. S-adenosylmethionine and S-adenosylhomocysteine are the most reliable biochemical markers. The major histological finding was pronounced microvesicular hepatic steatosis. Therapeutic trials with a methionine restricted diet indicate a potential beneficial effect on biochemical and clinical parameters in four patients and hyperinsulinism was responsive to diazoxide in two patients.

Conclusion Adenosine kinase deficiency is a severe inborn error at the cross-road of methionine and adenosine metabolism that mainly causes dysmorphism, brain and liver symptoms, but also recurrent hypoglycemia.
6

Institute of Pathology, University Hospital Heidelberg, Heidelberg, Germany

8 Genetics Research Center, University of Social Welfare and Rehabilitation Sciences, Tehran, Iran

9

Center for molecular diseases, CHUV Lausanne, Lausanne, Switzerland

10 Newborn Screening, Clinical Chemistry and Pharmacology Lab, NeuroFarba Department, Meyer Children's University Hospital, Florence, Italy

11 Center for Pediatrics and Adolescent Medicine, University Hospital Freiburg, Freiburg, Germany 
The clinical phenotype varies from an exclusively neurological to a multi-organ manifestation. Methioninerestricted diet should be considered as a therapeutic option.

\section{Introduction}

The essential amino acid methionine is next to protein synthesis involved in fundamental biological processes. The transmethylation of methionine to homocysteine is present in all mammalian cells and its disruption can lead to severe clinical phenotypes (Baric 2009). Defects of the single steps of the methionine cycle are MAT I/III deficiency (OMIM \#250850) (Chien et al 2015), GNMT deficiency (OMIM \#606664) (Mudd et al 2001) and SAHH deficiency (OMIM \#613752) (Baric et al 2004). Homocysteine and adenosine are the products of the hydrolysis of S-adenosylhomocysteine (AdoHcy). Cystathionine beta-synthetase (CBS) mediates the first step of the breakdown of homocysteine and causes classical homocystinuria if deficient (OMIM \#236200) (Mudd et al 1964). Adenosine kinase (ADK) converts adenosine to AMP, and a defective function of the enzyme leads to an accumulation of adenosine. This disrupts the methionine cycle by a reversal of the reaction balance of SAHH (Boison et al 2002, 2010). causing elevated concentrations of AdoHcy, S-adenosylmethionine (AdoMet) and methionine (Fig. 1). In 2011, Bjursell et al described the first six patients from three families with autosomal-recessive ADK deficiency (OMIM \#614300), that were characterized by global developmental retardation, epilepsy, liver dysfunction, dysmorphic features and hypermethioninemia (Bjursell et al 2011).

Adenosine is pivotal for maintenance of energy homeostasis in most organ systems including the brain (Newby et al 1985) and is an endogenous modulator of neuronal excitability (Dunwiddie 1980; Fredholm and Hedqvist 1980). AdoHcy is a powerful inhibitor of most methylation reactions that play a pivotal role in metabolism (Blom et al 2006). including epigenetic DNA methylation. ADK inhibitors and deficiency of ADK were shown to induce hypomethylation of DNA via biochemical interference with the transmethylation pathway in mice (Williams-Karnesky et al 2013). As may be expected from the importance of this pathway, autosomal-recessive ADK deficiency comprises a complex inborn error of metabolism with a severe clinical phenotype. So far, no specific therapeutic approach is known. This study of a greater number of individuals with ADK deficiency reveals new insights into this disease.

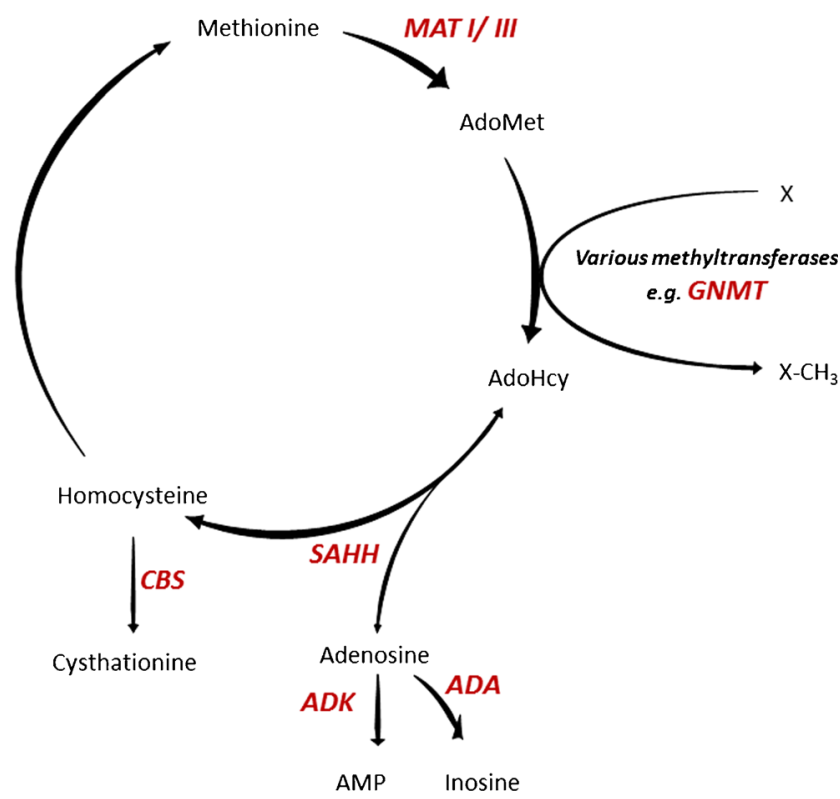

Fig. 1 Transmethylation of methionine to homocysteine and adenosine degradation. Via AdoMet and AdoHcy methionine is converted to homocysteine (Hcy), which can be remethylated back to methionine. AdoMet functions as donor of methyl-groups in a wide range of transmethylation reactions. SAHH hydrolyses AdoHcy into adenosine and Hcy. These products are under normal conditions constantly removed, preventing the accumulation of AdoHcy because the equilibrium of SAHH favours AdoHcy synthesis. There are two metabolic pathways that remove adenosine: the deamination into inosine via adenosine deaminase (ADA; EC 3.5.4.4) and the phosphorylation into AMP via adenosine kinase (ADK; EC 2.7.1.20). ADK has a low $\mathrm{Km}$ for adenosine, and is considered to be the main route of adenosine metabolism. In ADK deficiency, adenosine accumulates, reversing the reaction of SAHH to the condensation of AdoHcy. Via inhibition of transmethylation reactions, AdoHcy accumulation leads to an increase of AdoMet and finally methionine. Apart from ADK deficiency, deficiencies of MAT I/III, GNMT, SAHH and CBS are known inborn errors of metabolism of the transmethylation pathway. Deficiency of ADA causes severe combined immunodeficiency (SCID) (\#OMIM 102700). Adapted from The American Journal of Human Genetics, Vol 89, Magnus K. Bjursell, Henk J. Blom, et al, 507-515, 2007, with permission from Elsevier

\section{Materials and methods}

\section{Study design}

Clinical data of 11 patients from eight families identified with ADK deficiency were collected by a questionnaire, including anthropometrical data, first symptoms, clinical symptomatology, laboratory findings, as well as results of liver and muscle biopsies, and effects of dietary interventions. Written informed consent was obtained from all parents. For detailed case reports, see the Supplementary Appendix. Patients 9 and 10 have been identified to carry a homozygous mutation in $A D K$ through a large next generation sequencing study in individuals with intellectual disability (Najmabadi et al 2011). published practically at the same time (October 2011) as the 
initial description of ADK deficiency by Bjursell et al (Bjursell et al 2011). Apart from intellectual disability no details on the clinical course of the two patients have been published before. Material was not available for measurement of biochemical parameters (methionine, AdoMet and AdoHcy, total homocysteine, adenosine) in patients 9 and 10 .

\section{Liver histopathology}

Paraffin-embedded blocks of two liver biopsies of patient 2 were further analysed with immunohistochemistry and ultrastructural pathology. For immuno-histochemical analysis of steatosis, primary antibodies against lipid droplet-associated proteins of the perilipin family were used as described previously (Straub et al 2008; Pawella et al 2014). For ultrastructural pathology, biopsies were fixed in glutaraldehyde and embedded in epon. Thin sections were analysed in a transmission electron microscope (JEM 1400, JEOL).

\section{Measurements of AdoMet and AdoHcy in plasma and CSF and of adenosine in dried blood spots}

AdoMet and AdoHcy were measured in plasma and CSF via a stable-isotope dilution liquid chromatography-electrospray tandem mass spectrometry method, as published before (Gellekink et al 2005). Likewise, adenosine concentration in dried blood spots were measured via a stable-isotope dilution liquid chromatography-electrospray tandem mass spectrometry method, see reference (Azzari et al 2011) for details.

\section{Methionine loading test}

In patient 8, a methionine loading test was performed at the age of 7 years. Concentrations of methionine were determined in plasma before and 4, 6 and 12 hours after application of Lmethionine $(0.1 \mathrm{~g} / \mathrm{kg}$ body weight, oral administration).

\section{Results}

\section{Variable and extending clinical phenotype}

Since the first publication of ADK deficiency in 2011, we identified 11 new patients from eight families. Apart from family VII, all patients became symptomatic in the newborn period. First symptoms were mainly severe or prolonged conjugated hyperbilirubinaemia and hypoglycemia. In the course of the disease, muscular hypotonia and developmental delay were noticed in all individuals within the first year of life or shortly after. Severity of developmental delay varied from moderate to severe intellectual disability. Most patients developed epileptic seizures with onset (first afebrile seizure) between
4 months and 8.1 years (mean age of onset 27.4 months). EEG showed no significant abnormalities in the four patients without epilepsy. Whereas hyperbilirubinaemia was most prominent in the newborn period, all patients except patients 9 and 10 (family VII) had intermittent increases of transaminases and/or cholestasis in the further course, ranging from mild hepatopathy to liver failure. Hepatopathy tended to be less severe with increasing age. No trigger of liver crises was unambiguously identified. Some patients had intermittent hepatomegaly.

Nine patients had recurrent hypoglycemia, four already in the neonatal period. In three patients, hyperinsulinism was identified as the underlying cause, whereas it was not studied in most other cases. Two patients were treated with diazoxide with good response. Frontal bossing was present in all patients, whereas hypertelorism, sparse and thin hair, abnormal dentition, marfanoid features such as slender hands and feet, macrocephaly, short stature and failure to thrive were present in some. Five patients had cardiac defects, most of them were mild. Four patients developed cholelithiasis and three had megaloblastic anemia (see Table 1, Fig. 2 and in the Supplementary Appendix case reports and Table S1 for further details). Megaloblastic anemia could be due to reduced 5-methyltetrahydrofolate concentrations, possibly as a consequence of inhibition of methylentetrahydrofolate reductase activity due to the increased AdoMet concentrations. Muscle biopsy was performed in one patient (patient 2, biopsy at the age of 1 month) with normal results on light microscopy. There was no mitochondrial DNA depletion and pyruvate dehydrogenase complex activity was normal.

\section{Genotype}

In 11 patients from eight families, seven new mutations of the $A D K$ gene were identified (six families homozygous, one family compound heterozygous; see Table 1). Numbering is based on the short (cytosolic) isoform of $A D K$ (isoform a; transcript NM_001123.3; protein NP_001114.2). Types of mutations are as follows: deletion (1), frameshift (1), nonsense (2) and missense (3). All missense mutations are predicted to be possibly or probably damaging as predicted by "PolyPhen-2" (Score 0.87 - 1.0) (Adzhubei et al 2010). whereas all missense mutations are predicted to be disease causing using "Mutation taster" (Score 1.0) (Schwarz et al 2014). All mutations are extremely rare: none of the variants are listed in the 1000 Genome project, whereas one heterozygous allele carrier for c. $250 \mathrm{C}>\mathrm{T}$, p.Gln $84^{*}$ and two heterozygous allele carriers for c.953C $>$ A, p.Ala318Glu are listed in the Exome Aggregation Consortium (ExAC) Server (Cambridge, MA [10/2015]), that includes variants from $>120.000$ alleles of unrelated individuals. 


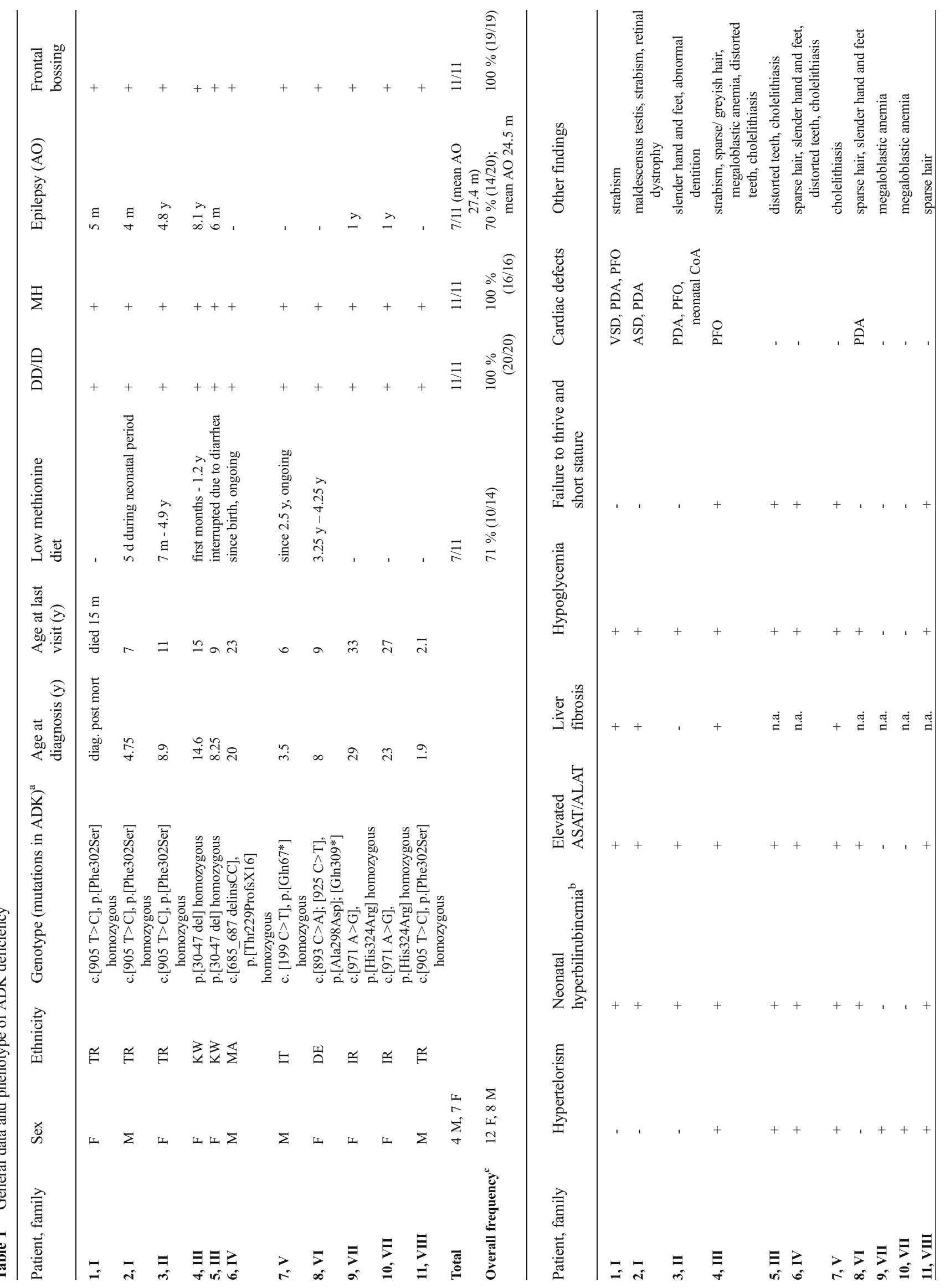




\section{Biochemical phenotype}

Metabolic workup revealed hypermethioninaemia in all patients. However, methionine levels in plasma vary and may even lie within the normal range (see Table 2 and Fig. S1). In contrast, concentrations of AdoMet and AdoHcy in plasma were always elevated, even when methionine concentrations were normal (e.g. patient 2 at the age of 6.8 years: methionine $42 \mu \mathrm{mol} / \mathrm{L}$ (reference range 15-45 $\mu \mathrm{mol} / \mathrm{L}$ ), AdoMet $212 \mathrm{nmol} / \mathrm{L}$ (reference range 71-118 nmol/L), AdoHcy $154 \mathrm{nmol} / \mathrm{L}$ (reference range $9.3-14 \mathrm{nmol} / \mathrm{L}$, all in plasma)). Adenosine concentrations in urine were only slightly elevated or in the upper normal range in some patients examined. In patient 2, adenosine concentrations varied from normal ( $1 \mathrm{mmol} / \mathrm{mol}$ creatinine) to clearly elevated $(22 \mathrm{mmol} / \mathrm{mol}$ creatinine, reference range $<4 \mathrm{mmol} / \mathrm{mol}$ creatinine) (see Table 2 for further details). Some patients have mildly elevated concentrations of total homocysteine (tHcy), which could be explained by decreased homocysteine remethylation due to inhibition of methylentetrahydrofolate reductase by AdoMet. Consistent or recurrent mild hyperammonaemia was described during the first months of life in patients 1 and 11, but has remained unexplained.

Methionine loading test in patient 7 revealed the following methionine concentrations in plasma: $0 \mathrm{~h}: 30 \mu \mathrm{mol} / \mathrm{L}$; $4 \mathrm{~h}$ : $441 \mu \mathrm{mol} / \mathrm{L} ; 6 \mathrm{~h}: 694 \mu \mathrm{mol} / \mathrm{L}$ and $12 \mathrm{~h}: 570 \mu \mathrm{mol} / \mathrm{L}$. In healthy subjects, methionine reaches it maximum between 0.5 to 1 hour after loading (Blom et al 1989, 1992). In contrast, in the ADK patient examined the peak was reached at 6 hours, which indicates a decreased uptake from the gut and/or delayed passage through the liver. The still greatly increased methionine concentration at 12 hours after loading points at a delayed conversion of excess methionine.

\section{Metabolites measured in newborn screening dried blood spots}

Methionine was measured in newborn screening DBS of three patients. Methionine concentration was normal in one case and mildly elevated in two cases $(96 \mu \mathrm{mol} / \mathrm{L}$ and $138 \mu \mathrm{mol} /$ L), but one of those two had a normal methionine concentration in the control sample seven days later (at the age of 10 days). Adenosine was measured in two newborn screening DBS and was clearly elevated in both (adenosine 3.78 and $3.82 \mu \mathrm{mol} / \mathrm{L}$, respectively; normal $<1.0$ ).

\section{Low methionine diet}

Seven patients received a low methionine diet consisting of a reduction of the daily intake of methionine to $15-20 \mathrm{mg}$ per $\mathrm{kg}$ of body weight. In patient 2 the diet was initiated during the newborn period but was discontinued after 5 days due to lack of clinical and biochemical response. Patient 5 did not tolerate 
Fig. 2 Dysmorpholgical symptoms in ADK deficiency. a-d Patient 3. a) Age 1.2 years. Icterus is evident, frontal bossing may be noticed. Hair is sparse and of abnormal texture. b-d) Age 4.25 years. Facial expression of a mentally retarded girl, frontal bossing is pronounced. Severe muscular hypotonia of head, trunk and limbs. e) Patient 6, aged 22 years. He has short stature $(151 \mathrm{~cm} /-4.5 \mathrm{SDS})$, macrocephaly (3.9 SDS) and has a long, trigonal face with frontal bossing. Teeth are distorted. Slender hands and fingers can be noted. f, g Patient 11. f) Age 14 months. Frontal bossing is prominent. g) Age 2.3 years. Relative macrocephaly, frontal bossing and muscular hypotonia
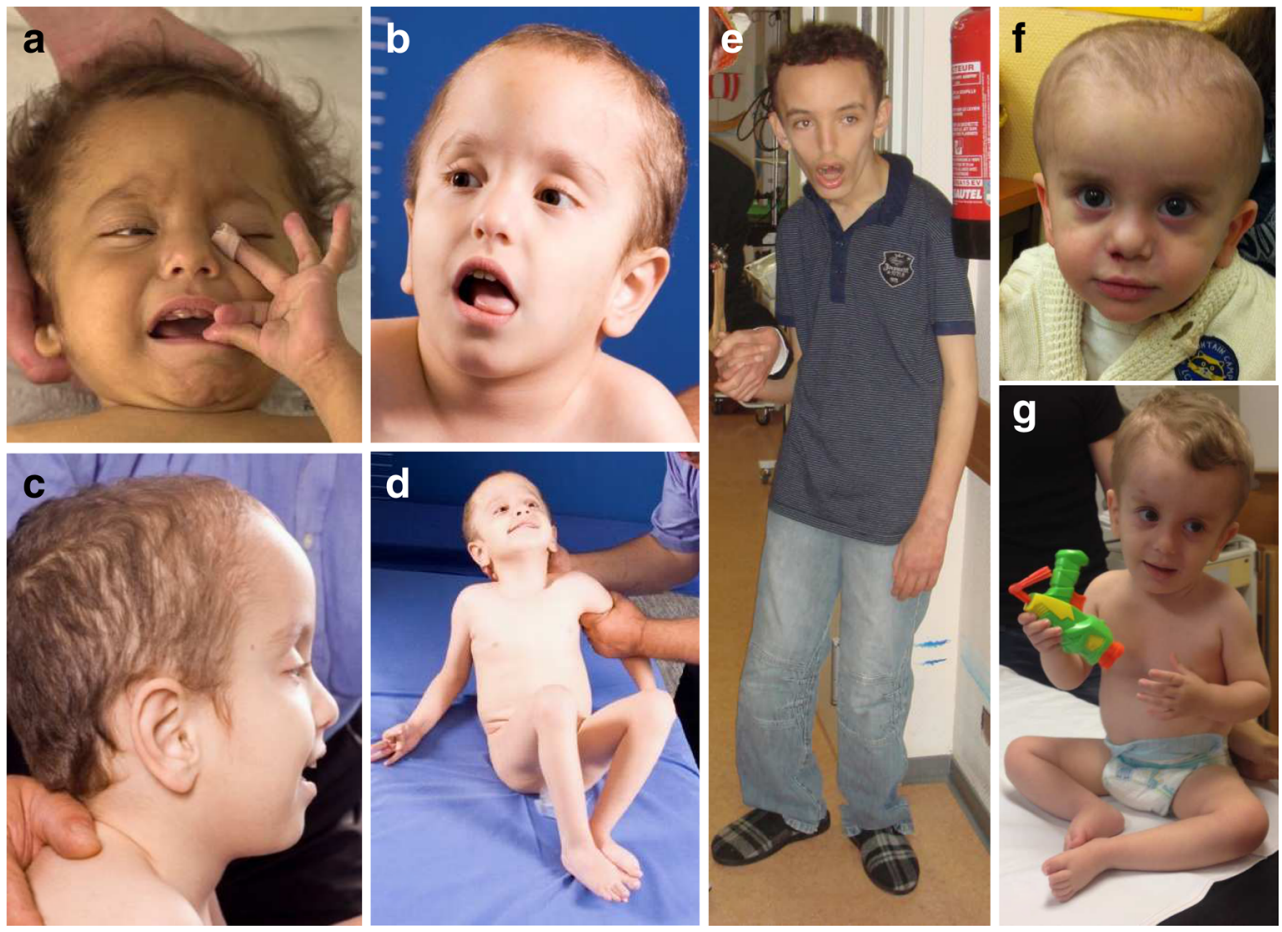

methionine free formula as she developed intractable diarrhoea even with changing the diet to a different type of methionine free formula, and the parents decided to stop the dietary treatment. In patient 8 the diet was stopped after one year (at the age of 4.25 years) because it was considered ineffective. In contrast, patients 3, 4, 6 and 7 showed clear improvement of clinical and/or biochemical parameters after the start of a low methionine diet. Patient 6 has been treated with a low methionine diet since birth. Discontinuation of the diet resulted in acute deterioration of liver function at the age of 20 years, which was reversible after reintroduction of dietary treatment. Also in patients 3 and 7 improvement of liver function was noted (see Fig. S1 and Table S2) and in addition, the frequency of hypoglycaemic episodes decreased. In patient 7 , moreover a striking improvement of the neurological phenotype with amelioration of developmental delay, motor function and speech was observed. In all patients (except patient 2, who was only treated for 5 days) a clear biochemical response with decrease or normalization of methionine levels and decrease of AdoMet and AdoHcy concentrations in plasma was noted under therapy. In addition, in patient 7 a decrease of adenosine concentration in DBS was observed (see Table S2).

\section{Liver biopsies}

Liver biopsies were taken from five patients. Signs of cholestasis were described (2 patients), a reduced number of bile ducts (2) and mild lobular hepatitis (1). Four patients showed portal or periportal fibrosis (already observed at the age of 1 month in patient 1). In one patient, paraffin blocks of two liver biopsies were available for further immunohistochemical and ultrastructural studies. Immunohistochemistry for perilipins, markers for hepatocyte steatosis, demonstrated pronounced long-standing microvesicular steatosis as well as some ballooned cells as a sign of steatohepatitis. In transmission electron microscopy, numerous small lipid droplets and large areas of glycogen deposits were detected as well as some polymorphic lysosomes (Fig. 3).

\section{Discussion}

This study describes the clinical phenotype of 11 newly identified patients with ADK deficiency from eight families. Hitherto this disease has been described in six patients from three families. Together with those previous observations our report significantly enlarges and defines the complex clinical findings of this disease, which is likely to remain undiagnosed in many cases because of the different and varying facets. All patients share the clinical features of psychomotor retardation, muscular hypotonia and frontal bossing. All but one family presented with hepatic involvement that manifested as prolonged or severe hyperbilirubinaemia in the neonatal period and recurrent liver crises with elevated transaminases and/ or cholestasis in the further course, which were most severe in the first year of life. The best markers for laboratory diagnosis appear to be elevations of AdoMet and AdoHcy in plasma, the investigation of which is unfortunately only performed in very few specialized metabolic laboratories in the world. 


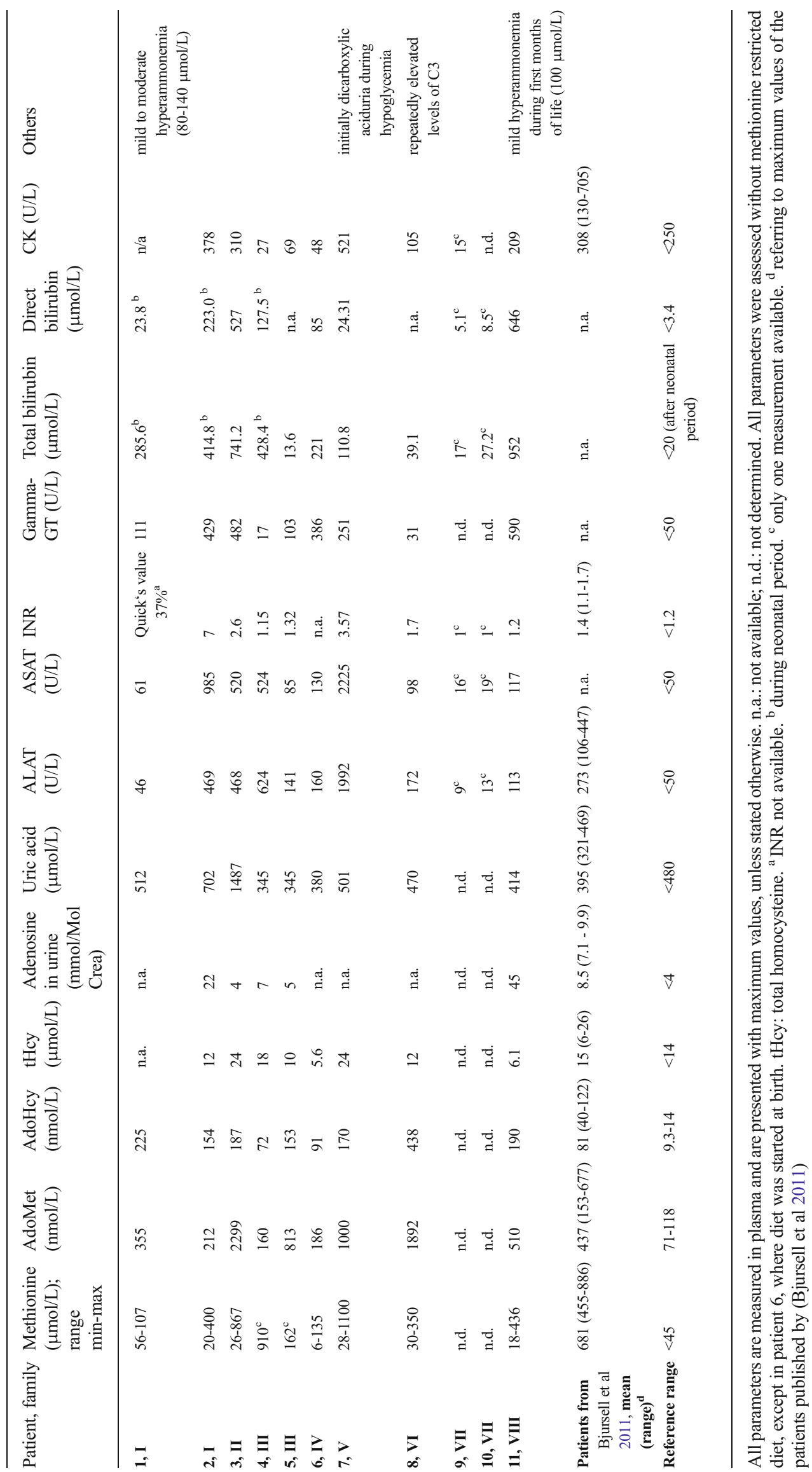


Fig. 3 Representative histology and ultrastructure of liver biopsies. Liver biopsy of patient 2 revealed microvesicular steatosis, chronic cholestasis and portal, periportal and perisinusoidal fibrosis $(\mathbf{a}, \mathbf{b})$. c, $\mathbf{d}$ : Pronounced acute and chronic microvesicular steatosis was demonstrated by immunohistochemical staining against perilipins 1 and 2 (plin1, plin2). e, f: In electron microscopy, hepatocytes showed large pleomorphic lysosomes, and small-sized cytoplasmic and nuclear lipid inclusions. Abbreviations: H\&E: hematoxylin\&eosin-stain, TEM: transmission electron microscopy, PT: portal tract, emh: extramedullary haematopoiesis, HSC: hepatic stellate cell, LSEC: liver sinusoidal endothelial cell; LD(s): lipid droplet(s), ery: erythrocyte; rER: rough endoplasmic reticulum, bc: ballooned cell. Bars: each indicated in right lower corner
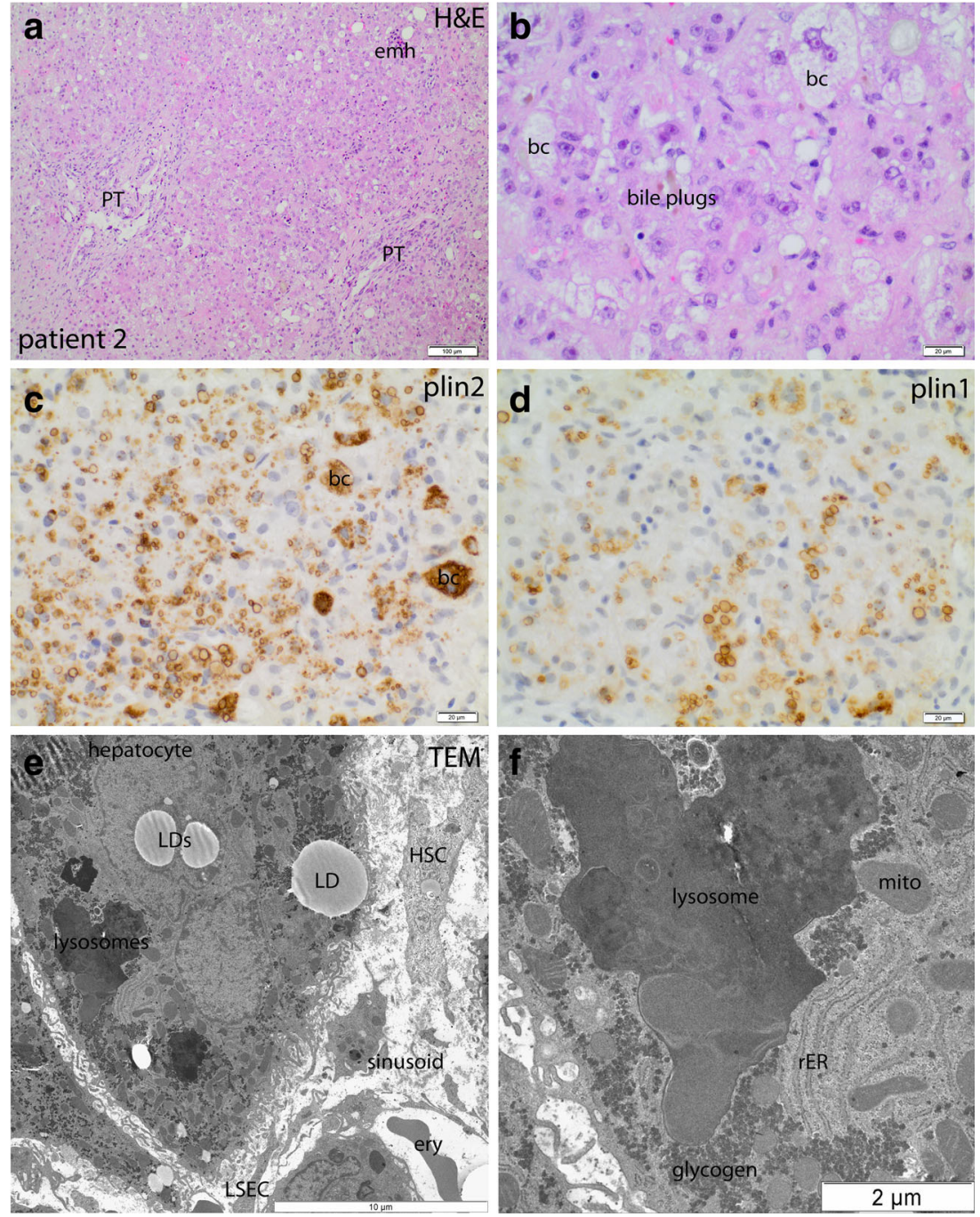

\section{Neurological phenotype}

The patients described by Bjursell et al (Bjursell et al 2011) developed epilepsy early in the disease course (mean age 18.2 months, range 10 - 35). In our study, age at onset varied between 4 months to 8.1 years, and some patients have remained seizure free until now. Thus, epilepsy is frequent in ADK deficiency but is more variable than initially described. The mechanism of brain damage in ADK deficiency is unclear; however, it is likely to involve lack of energy metabolites (ATP), inhibition of transmethylation reactions like DNA and protein methylation and alteration of adenosine signalling via adenosine receptors. Adenosine is an endogenous modulator of neuronal excitability (Dunwiddie 1980) and through adenosine receptors (A1, A2A, A2B, A3), which partly interact with other $\mathrm{G}$ protein-coupled receptors, adenosine affects all major neurotransmitter and neurotrophin systems (Sebastiao and Ribeiro 2009). Since the last decade, adenosine has been considered an endogenous anticonvulsant and neuroprotectant (Boison et al 2010). Adenosine kinase overexpression (e.g. due to astrogliosis) and, as a consequence, adenosine depletion were shown to be epileptogenic, whereas a surge of adenosine through trauma or hypoxia induced ADK deficiency was neuroprotective (Boison 2012). Adenosine augmentation therapy (through inhibition of adenosine kinase or delivery of adenosine) is seen as a powerful pharmacological approach for treatment of epilepsy (Boison 2012; Theofilas et al 2011). The high rate of epilepsy among patients with ADK deficiency however additionally suggests an epileptogenic potential of increased adenosine concentrations. Patients with SAHH deficiency do not develop epilepsy (Baric et al 2004, 2005). which underscores a pathogenic role of adenosine for epilepsy in ADK deficiency. Maintaining a balanced system of adenosine signalling should be kept in mind for further approaches of adenosine augmentation therapy.

As creatine synthesis depends on AdoMet-mediated methylation and accounts for a about $40 \%$ of whole body AdoMet 
(Brosnan et al 2011). cerebral creatine deficiency may be considered as another player contributing to brain damage in ADK deficiency.

\section{Liver phenotype}

Liver dysfunction in ADK deficiency varies from mild hyperbilirubinaemia or mildly elevated transaminases to frank liver failure. Similar to citrine deficiency (OMIM \#605814), Niemann Pick type C (OMIM \#257220) or mannosephosphate isomerase deficiency (MPI-CDG, formally CDG-Ib, OMIM \#602579), ADK deficiency should be included in the metabolic differential diagnosis of transient neonatal cholestasis. Fatal neonatal liver steatosis has been described in an ADK deficient mouse model (Boison et al 2002). In addition to steatosis, liver biopsy of patient 2 revealed subsequent liver damage with ballooned cells and mild inflammation indicative of steatohepatitis, with portal and periportal fibrosis and chronic cholestasis. Fatty acids are a major source of energy in the neonate and mitochondrial function becomes critical for energy metabolism after birth (Chalmers et al 1997; Boison 2012). and microvesicular steatosis may indicate mitochondrial dysfunction and deficiencies in fatty acid oxidation (Burt et al 1998; Day and James 1998). In the mouse model, decreased concentrations of the adenine nucleotides AMP, ADP and ATP were measured in liver tissue homogenates, suggesting energy deficiency as a possible mechanism for liver steatosis in ADK deficiency. Reduced methylation through AdoHcy mediated inhibition of transmethylation reactions was discussed as another mechanism (Boison et al 2002). The clearly elevated concentrations of AdoMet likely indicate an inhibition of transmethylation reactions in our patients.

\section{Hypoglycemia}

Hypoglycemia has not been reported as a clinical sign of ADK deficiency before. However, in our patients recurrent and/or severe hypoglycemia was one of the first and most common symptoms. In some patients, hyperinsulinism could be detected as the cause of recurrent hypoglycemia. Inhibitors of ADK and adenosine agonists were recently shown to specifically promote replication of primary $\beta$-cells of the pancreas in vitro and in vivo through the adenosine receptor A2aa and activation of the mTOR pathway (Annes et al 2012; Andersson et al 2012). Our finding of hyperinsulinism in patients with ADK deficiency suggests that adenosine signalling may influence insulin secretion in humans. Increased glycogen deposits as seen in the liver biopsies studied may point to increased glycogen storage (i.e. as consequence of hyperinsulinsim), but other effects such as impairment of glycogenolysis cannot be ruled out.

Patient 7 initially presented a picture similar to a defect of fatty acid oxidation with dicarboxylic aciduria and unresponsiveness to glucagon, before he developed hyperinsulinism. Hence, mechanisms of hypoglycemia in ADK deficiency may be multilayered.

\section{Incidence and diagnosis}

The incidence of ADK deficiency is unknown. Literature review of unexplained cases of hypermethioninaemia revealed the three siblings published by Labrune et al in 1990 (Labrune et al 1990). that finally proved to be sisters of one of our patients (see case report family IV in the Supplementary Appendix). Genetic confirmation has not been done in the three older sisters, but as they show a typical clinical and biochemical picture and are siblings of a genetically confirmed case of ADK deficiency, this "familial hypermethioninemia" can be considered to be ADK deficiency. Thus up to now, including the patients published by Bjursell et al, Labrune et al (Bjursell et al 2011; Labrune et al 1990) and the patients of this publication, 20 individuals with ADK deficiency are known, of whom 18 are alive at time of report. We expect ADK deficiency to be an underdiagnosed disease for several reasons: the disease is only known to a few specialists, it has a wide range of clinical phenotypes and most biochemical findings can be intermittent, especially hypermethioninaemia, and measurement of the most sensitive parameters AdoMet and AdoHcy is only performed in very few specialized metabolic laboratories. It is likely that so far the severe end of the phenotype has been described and family VIII suggests that there are patients with ADK deficiency among individuals with intellectual disability without other organ manifestations. Ethnical diversity of our patients underlines that this disease is not a regional phenomenon.

ADK deficiency is characterized by the pattern of elevated concentrations of methionine, AdoMet and AdoHcy in plasma and adenosine in urine. However, concentrations of methionine and adenosine can be normal. Measurement of AdoMet and AdoHcy in plasma appears to be more sensitive than methionine in plasma, as AdoMet has been elevated at all times in our patients (also in the initially described patients). AdoHcy also has been elevated in all our patients, but was normal in one of the patients described by Bjursell (Bjursell et al 2011). Hypermethioninemia is often secondary to liver disease. However, if there is isolated hypermethioninemia or when there is a clinical suspicion of ADK deficiency, AdoMet and AdoHcy should be measured for further differentiation. Adenosine in dried blood spots as a potential biomarker in newborn screening requires further study.

\section{Therapeutic approach}

Taking into account the three patients that were published by Labrune et al (Labrune et al 1990). there is evidence for a positive effect of a low methionine diet in ADK deficiency in seven patients from different centres while there are no reports 
of adverse side effects apart from diarrhoea in one patient. Especially the liver phenotype seems to improve, whereas a positive effect on the neurological outcome has been reported in a single case so far. Decreased intake of methionine resulted in a clear biochemical response as measured in blood (Table S2 and Fig. S1). It is tentative to speculate that the dietary intervention may also normalize AdoMet and AdoHcy in CSF. The patients described by Bjursell et al had elevated concentrations of AdoMet and AdoHcy in CSF without diet (Bjursell et al 2011) whereas patient 7 of this study had normal concentrations of AdoMet and AdoHcy in CSF on diet; however, these concentrations have not been assessed before start of the diet in patient 7 . The mechanism of the effect of the low methionine diet remains to be studied but it seems reasonable that reduced abnormalities of the key metabolites adenosine, AdoHcy and AdoMet will have a positive effect on the disturbed metabolism in the disorder. The murine ADK deficient model displayed reduced ADP and ATP concentrations, and the implications of a reduction of adenosine on these cellular key-metabolites are hard to predict. An alternative approach to reduce adenosine levels would be through activating alternative pathways for its degradation. A recombinant ADA enzyme supportive therapy (already available for ADA-SCID patients) may be a future therapy option for patients with ADK deficiency and should be evaluated in the murine ADK deficient model.

Experience gathered so far indicates that diazoxide is an effective treatment of recurrent hypoglycemia caused by hyperinsulinism in ADK deficiency.

Here we present 11 new patients from eight families with ADK deficiency, which were identified since the first description of the disorder in 2011. The facets and spectrum of disease are defined, and ADK deficiency should be suspected in infants with (transient) cholestatic liver disease and muscular hypotonia, epilepsy or other neurological symptoms, especially when there is also recurrent hypoglycemia and frontal bossing. However, the absence of hepatic or metabolic symptoms does not rule out ADK deficiency. Hypermethioninaemia, increased AdoMet and AdoHcy concentrations in plasma and increased adenosine concentration in urine point to ADK deficiency biochemically. As plasma methionine and adenosine in urine can be normal, measurement of AdoMet and AdoHcy is of special importance. Confirmatory diagnostics can be achieved through molecular genetics of the $A D K$ gene.

A low methionine diet should be considered as a therapeutic option as most patients showed an improvement of hepatopathy and as there is a potential beneficial effect on neurological outcome, whereas diazoxide is effective to treat hyperinsulinism. Prospective registries such as E-HOD (http://www.e-hod.org) will give further insight into the natural history of this most interesting inborn error of metabolism, which affects both the methionine cycle and adenosine metabolism.
Acknowledgments We especially thank Zlata Antoni, Elisabeth Specht and Johanna Kugele for excellent technical assistance.

\section{Compliance with Ethical Standards}

Funding Part of the study was funded by grants of the German Research Foundation to BKS (DFG STR1160/1-1 and 1-2).

\section{Conflict of interest None.}

Informed consent All procedures followed were in accordance with the ethical standards of the responsible committee on human experimentation (institutional and national) and with the Helsinki Declaration of 1975 , as revised in 2000. Informed consent was obtained from all patients or their parents in case of minor patients for being included in the study. Additional informed consent was obtained from all patients for whom identifying information is included in this article.

\section{References}

Adzhubei IA, Schmidt S, Peshkin L et al (2010) A method and server for predicting damaging missense mutations. Nat Methods 7(4):248249

Andersson O, Adams BA, Yoo D et al (2012) Adenosine signaling promotes regeneration of pancreatic beta cells in vivo. Cell Metab 15(6):885-894

Annes JP, Ryu JH, Lam K et al (2012) Adenosine kinase inhibition selectively promotes rodent and porcine islet beta-cell replication. Proc Natl Acad Sci U S A 109(10):3915-3920

Azzari C, la Marca G, Resti M (2011) Neonatal screening for severe combined immunodeficiency caused by an adenosine deaminase defect: a reliable and inexpensive method using tandem mass spectrometry. J Allergy Clin Immunol 127(6):1394-1399

Baric I (2009) Inherited disorders in the conversion of methionine to homocysteine. J Inherit Metab Dis 32(4):459-471

Baric I, Fumic K, Glenn B et al (2004) S-adenosylhomocysteine hydrolase deficiency in a human: a genetic disorder of methionine metabolism. Proc Natl Acad Sci U S A 101(12):4234-4239

Baric I, Cuk M, Fumic K et al (2005) S-Adenosylhomocysteine hydrolase deficiency: a second patient, the younger brother of the index patient, and outcomes during therapy. J Inherit Metab Dis 28(6):885902

Bjursell MK, Blom HJ, Cayuela JA et al (2011) Adenosine kinase deficiency disrupts the methionine cycle and causes hypermethioninemia, encephalopathy, and abnormal liver function. Am J Hum Genet 89(4):507-515

Blom HJ, Boers GH, Trijbels JM, van Roessel JJ, Tangerman A (1989) Cystathionine-synthase-deficient patients do not use the transamination pathway of methionine to reduce hypermethioninemia and homocystinemia. Metab Clin Exp 38(6):577-582

Blom HJ, Davidson AJ, Finkelstein JD et al (1992) Persistent hypermethioninaemia with dominant inheritance. J Inherit Metab Dis 15(2):188-197

Blom HJ, Shaw GM, den Heijer M, Finnell RH (2006) Neural tube defects and folate: case far from closed. Nat Rev Neurosci 7(9): $724-731$

Boison D (2012) Adenosine dysfunction in epilepsy. Glia 60(8):1234 1243

Boison D, Scheurer L, Zumsteg V et al (2002) Neonatal hepatic steatosis by disruption of the adenosine kinase gene. Proc Natl Acad Sci U S A 99(10):6985-6990 
Boison D, Chen JF, Fredholm BB (2010) Adenosine signaling and function in glial cells. Cell Death Differ 17(7):1071-1082

Brosnan JT, da Silva RP, Brosnan ME (2011) The metabolic burden of creatine synthesis. Amino Acids 40(5):1325-1331

Burt AD, Mutton A, Day CP (1998) Diagnosis and interpretation of steatosis and steatohepatitis. Semin Diagn Pathol 15(4):246-258

Chalmers RA, Stanley CA, English N, Wigglesworth JS (1997) Mitochondrial carnitine-acylcarnitine translocase deficiency presenting as sudden neonatal death. J Pediatr 131(2):220-225

Chien YH, Abdenur JE, Baronio F et al (2015) Mudd's disease (MAT I/III deficiency): a survey of data for MAT1A homozygotes and compound heterozygotes. Orphanet J Rare Dis 10:99

Day CP, James OF (1998) Hepatic steatosis: innocent bystander or guilty party? Hepatology 27(6):1463-1466

Dunwiddie TV (1980) Endogenously released adenosine regulates excitability in the in vitro hippocampus. Epilepsia 21(5):541-548

Fredholm BB, Hedqvist P (1980) Modulation of neurotransmission by purine nucleotides and nucleosides. Biochem Pharmacol 29(12):1635-1643

Gellekink H, van Oppenraaij-Emmerzaal D, van Rooij A, Struys EA, den Heijer M, Blom HJ (2005) Stable-isotope dilution liquid chromatography-electrospray injection tandem mass spectrometry method for fast, selective measurement of S-adenosylmethionine and S-adenosylhomocysteine in plasma. Clin Chem 51(8):1487-1492

Labrune P, Perignon JL, Rault M et al (1990) Familial hypermethioninemia partially responsive to dietary restriction. J Pediatr 117(2 Pt 1):220-226

Mudd SH, Finkelstein JD, Irreverre F, Laster L (1964) Homocystinuria: an enzymatic defect. Science 143(3613):1443-1445
Mudd SH, Cerone R, Schiaffino MC et al (2001) Glycine Nmethyltransferase deficiency: a novel inborn error causing persistent isolated hypermethioninaemia. J Inherit Metab Dis 24(4):448-464

Najmabadi H, Hu H, Garshasbi M et al (2011) Deep sequencing reveals 50 novel genes for recessive cognitive disorders. Nature 478(7367): $57-63$

Newby AC, Worku Y, Holmquist CA (1985) Adenosine formation. Evidence for a direct biochemical link with energy metabolism. Adv Myocardiol 6:273-284

Pawella LM, Hashani M, Eiteneuer E et al (2014) Perilipin discerns chronic from acute hepatocellular steatosis. J Hepatol 60(3):633642

Schwarz JM, Cooper DN, Schuelke M, Seelow D (2014) MutationTaster2: mutation prediction for the deep-sequencing age. Nat Methods 11(4):361-362

Sebastiao AM, Ribeiro JA (2009) Tuning and fine-tuning of synapses with adenosine. Curr Neuropharmacol 7(3):180-194

Straub BK, Stoeffel P, Heid H, Zimbelmann R, Schirmacher P (2008) Differential pattern of lipid droplet-associated proteins and de novo perilipin expression in hepatocyte steatogenesis. Hepatology 47(6): 1936-1946

Theofilas P, Brar S, Stewart KA et al (2011) Adenosine kinase as a target for therapeutic antisense strategies in epilepsy. Epilepsia 52(3):589 601

Williams-Karnesky RL, Sandau US, Lusardi TA et al (2013) Epigenetic changes induced by adenosine augmentation therapy prevent epileptogenesis. J Clin Invest 123(8):3552-3563 